\title{
An Adaptive, Region-based Allocator for Java
}

\author{
Feng Qian \\ Laurie Hendren \\ School of Computer Science, McGill University \\ 3480 University Street, Montreal, Quebec \\ Canada H3A 2 A7 \\ \{fqian, hendren\}@cs.mcgill.ca
}

\begin{abstract}
This paper introduces an adaptive, region-based allocator for Java. The basic idea is to allocate non-escaping objects in local regions, which are allocated and freed in conjunction with their associated stack frames. By releasing memory associated with these stack frames, the burden on the garbage collector is reduced, possibly resulting in fewer collections.

The novelty of our approach is that it does not require static escape analysis, programmer annotations, or special type systems. The approach is transparent to the Java programmer and relatively simple to add to an existing JVM. The system starts by assuming that all allocated objects are local to their stack region, and then catches escaping objects via write barriers. When an object is caught escaping, its associated allocation site is marked as a non-local site, so that subsequent allocations will be put directly in the global region. Thus, as execution proceeds, only those allocation sites that are likely to produce non-escaping objects are allocated to their local stack region.

The paper presents the overall idea, and then provides details of a specific design and implementation. In particular, we present a region-based allocator and the necessary modifications of the Jikes RVM baseline JIT and a copying collector. Our experimental study evaluates the idea using the SPEC JVM98 benchmarks, plus one other large benchmark. We show that a region-based allocator is a reasonable choice, that overheads can be kept low, and that the adaptive system is successful at finding local regions that contain no escaping objects.
\end{abstract}

\section{Categories and Subject Descriptors}

D.3.4 [Programming Languages]: Processors-Memory management (garbage collection)

\section{General Terms}

Languages, Performance, Experimentation

Permission to make digital or hard copies of all or part of this work for personal or classroom use is granted without fee provided that copies are not made or distributed for profit or commercial advantage and that copies bear this notice and the full citation on the first page. To copy otherwise, to republish, to post on servers or to redistribute to lists, requires prior specific permission and/or a fee.

ISMM'02, June 20-21, 2002, Berlin, Germany.

Copyright 2002 ACM 1-58113-539-4/02/0006 ...\$5.00.

\section{INTRODUCTION}

The Java [2] programming language implicitly uses a garbage collector as its memory manager. Garbage collection has the advantage of freeing memory safely and often precisely. However, tracing and collecting objects adds overhead to the program, so reducing the frequency of garbage collection can be beneficial. Stack-allocation of objects is one promising approach to reduce the work of a garbage collector [8]. If an object does not escape a method, it can be created on the stack frame instead of in the heap. Objects on the stack can be reclaimed without intervention by the garbage collector.

There are several potential obstacles to performing object stack-allocation in a JVM. Existing stack-allocation techniques require a static escape analysis $[5,4,16]$, which usually requires a whole program analysis and is not easily amenable to a JVM with dynamic class loading and JIT compilation. Further, as discussed by Gay and Steensgaard [8], there are several restrictions on the kinds of objects that can be created on the stack. Objects with non-trivial finalize methods are not easily placed on the stack, arrays may be too large, and objects created in a loop with overlapping lifetimes are not stackable.

To address these issues in a JVM, we suggest an adaptive region-based allocator. In our approach we treat local regions as extensions of stack frames. Thus, stackable objects can be created in regions instead of stack frames without the above restrictions. If a region contains only non-escaping objects, then when its associated stack frame is popped, the region can be deallocated by returning its associated pages to the free list of the heap.

Since we wish to avoid potentially expensive static analyses, we instead take the approach of an adaptive strategy which dynamically categorizes allocation sites as local and non-local. Initially objects are assumed to be local and the $\mathrm{VM}$ relies on write barriers to detect escaping objects. When an object escapes, two actions are taken. First, the corresponding region is marked as dirty. Second, the allocation site of the escaping object is categorized as non-local, so that subsequent allocations from that site will be put directly in a global region. A dirty region is deallocated by appending the pages associated with that region to the global region waiting for the next garbage collection, while the pages of a clean region can be reclaimed immediately. As execution proceeds, more allocation sites creating escaping objects are marked as non-local, so that local regions are more likely to stay clean.

Our approach requires a region-based allocator as its ba- 
sis. In general, heaps organized as pages/chunks provide a flexible way to divide memory into regions for different purposes, for example, regions [14, 13, 6] or thread-specific heaps [12]. However, the maintenance of regions and unused space on pages can lead to extra costs and overheads when compared to a flat heap organization. Thus, we first outline the design of a region-based allocator and we show the behavior of Java programs on page-based heaps (i.e., heaps consisting of a set of chunks/pages). Our study shows that most allocations can be satisfied with inexpensive operations and that the correct choice of page size leads to little wasted space. This suggests that the page-based heap organization is feasible for Java VMs.

We then show how to build the adaptive system for categorizing allocations into those which should be allocated in local regions associated with stack frames, and those that should be directly allocated in the global region. In the design we carefully consider the overheads, both in space and extra write barrier overhead, and we propose various approaches for keeping these overheads reasonably small.

This paper makes the following contributions:

1. We quantitatively studied the allocation behaviors of Java programs in a region-based allocator. This study indicates that dividing heap into special regions is feasible for Java programs.

2. In contrast to previous work, our memory manager does not require expensive static analysis, programmer directives, or special type systems. It is completely transparent to the Java programmer, and requires relatively few modifications to the JIT compiler.

3. Our method focuses on the allocation of new objects and can work as an optimization in conjunction with other GC algorithms - we have implemented it in conjunction with an extant type-accurate copying collector. Our method also works on Java in its full generality, including multiple threads, dynamic class loading and the JNI.

4. We have carefully analyzed the overheads of our approach and we suggest several mechanisms for reducing this overhead. We use lazy region allocation to reduce the number of region allocations and deallocations. Further, even though our method requires some extra state in the object header, we show how to implement this in a standard two-word header with a small tradeoff in the performance of thin locks.

We have implemented the proposed approach in the Jikes $\mathrm{RVM}^{1}$ [1] and have performed our initial study using its baseline compiler. This allows us to evaluate the approach's behavior and potential. We are currently working on extending the implementation to the optimizing compiler where we can evaluate further runtime speedups.

The paper is organized as follows. In Section 2, we introduce the overall structure of the system. The design and implementation of the allocator are presented in Section 3. Sections 4 and 5 describe the required modifications to the JVM and collector to utilize the new functionalities provided by the allocator. In Section 6, we present experimental results that focus on the behavior of the approach. Finally,

\footnotetext{
${ }^{1}$ formerly known as the Japapeño Virtual Machine.
}

we discuss the related work in Section 7 and conclusions and future improvements in Section 8.

\section{OVERVIEW}

The whole system consists of three parts: the allocator manages regions and allocates space for objects; the JIT compiler inserts instructions for acquiring and releasing a region in each compiled method; and the collector performs garbage collection when no more heap space is available.

In a region system, the heap space is divided into pages. The pages can be fixed-size or variable-size. In our system, we use fixed-size pages for fast computation of page numbers from addresses. The allocator is also a region manager. It manages a limited number of tokens. Each token is a small integer number identifying a region. Two regions, GLOBAL and FREE, exist throughout the execution of a program. Other, local regions, exist for shorter durations. They are assigned to and released by methods dynamically.

A high-level view of our memory organization is given in Figure 1. A more detailed description of the implementation is given in Section 3 .

A region token points to a list of pages in the heap. The region space is expanded by inserting free pages at the head of the list. The GLOBAL region contains objects created by non-local allocation sites and pages containing objects that have escaped out of local regions. The GLOBAL region space can only be reclaimed by the collector. The system uses bit maps to keep track of free pages in the heap. The pages of a local region can be appended to the GLOBAL region or reclaimed by resetting their entries in the bit map.

A method activation obtains a region token by either acquiring an available token from the region manager or by inheriting its caller's token. The region identified by the token acts as an extension of the activation's stack frame. Before exiting, the activation releases the region if it was not inherited. It is clear that the lifetime of a local region is bounded by the lifetime of its host stack frame. There is a many-to-one mapping between stack frames and regions.

An object can be created in the region of the top stack frame or in the GLOBAL region. For the remainder of the discussion we need to define what we mean by an object escaping from a region, and a non-local allocation site.

DEFINITION 1. An object escapes its allocation region if and only if it becomes pointed to by an object in another region.

Definition 2. An allocation site becomes non-local when an object created by that site escapes.

Given this definition of escape, there are only three Java bytecode instructions, putstatic, putfield, and aastore, that can lead to an object escaping. Therefore, it is sufficient to insert write barriers before these instructions to detect the escape of an object.

There is one additional situation that must be considered. When a method returns an object, the object may escape its allocation region via stack frames. However, this kind of escape can be prevented by either: (1) inserting write barriers before all areturn byte codes, or (2) requiring all methods returning objects to inherit their caller's region. In our implementation we have taken the second approach. It should be noted that objects passed to the callee as parameters are 


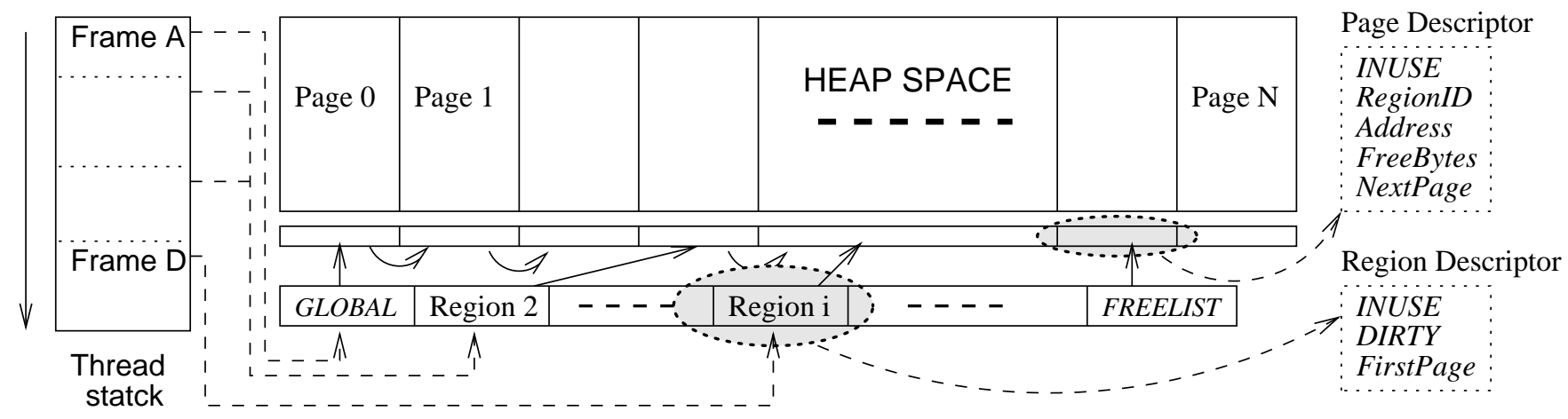

Figure 1: Memory organization of page-based heaps with regions

not a problem since the lifetime of the callee's stack frame is bounded by the caller's.

For an assignment such as lhs. $f=r h s$, the write barrier checks if the rhs is in the same region as the lhs object. When they are in different regions, the region containing the $r h s$ object is marked as dirty. Since static fields are much like global variables, we assume that a putstatic always leads to the rhs object escaping, and the region associated with this object is marked as dirty.

It is worth pointing out that a region cannot contain an object reachable from other regions without being marked as dirty. If there is a path which causes an object $o_{1}$ of a region $R_{1}$ to be reached from objects in other regions, there must be an object, say $o_{i}$, in $R_{1}$ which is on the path and is directly pointed to by another object not in $R_{1}$, and the assignment of this pointer must have been captured by write barriers. Hence, $R_{1}$ must be marked as dirty when such a path exists.

Each allocation site in a compiled method is uniquely indexed, and each object has a field in its header for recording the index of its allocation site (see Section 4 for a discussion of how this is accomplished without increasing the object header size). The allocator maintains a bit vector to record the states of the allocation sites. Besides marking the region dirty, the write barrier also marks an escaping object's allocation site as non-local. The allocator allocates objects in local regions only for local allocation sites. By not allocating objects for non-local sites in the local region, future activations of the method are very likely to have a local region containing only non-escaping objects.

The system is quite straightforward and we have implemented it in the Jikes RVM [1]. The Jikes RVM is written in Java. It has a baseline compiler which generates machine code simulating a stack machine. It also has an optimizing compiler which uses many sophisticated optimizations. The prototype of the allocator is implemented with the baseline compiler only. When we present the VM-related part in Section 4 , the stack frame layout refers to the conventions of the baseline compiler.

\section{ALLOCATOR}

\subsection{Heap organization}

Various garbage collection techniques have different heap organizations. For example, mark-and-swap collectors use a single space, copying collectors divide space into two semispaces, and generational collectors divide the heap into sev- eral aging areas. In this paper, the heap we are discussing refers to the space where new objects are allocated.

A region memory manager organizes a heap as pages. Without loss of generality, the heap in our system is organized as contiguous pages with a fixed size which is a power of 2 . The starting address of the heap is aligned to the page size. Therefore, computing the page number for an address requires only subtraction and bit shifting. Some systems do not allocate the large objects from regions, and do not allow an object to straddle two pages. In order to get a full picture of allocation behaviors, our system does not use a separate space for large objects and attempts to allocate objects on contiguous pages whenever it can.

Figure 1 gives a high-level overview of the memory organization that we use for our implementation of regions. A page descriptor encodes page status, region identification, allocation point, and the index of next page. A region descriptor contains region status, and the first page index of the region.

This organization provides sufficient information for regionbased allocation. When allocating space in a region, the allocator first checks the free bytes of the first page. When there is not enough space left there, a free page is taken from the free list and inserted in the page list as the first page. Allocating space for large objects involves searching for contiguous free pages. We have measured the overhead for these allocations for our benchmarks, and as shown in Section 6 , the frequency of expensive searches is quite low, indicating that this is a reasonable design.

\subsection{Services}

The internal heap organization is transparent to the JVM. The allocator provides a set of services to the JVM and collector. We describe these functions here.

There are two services for region operations as shown in Figure 2. Internally, free region tokens are managed by a stack. The NEWREGION service pops a region token from the stack, and pre-allocates one free page for it (pre-allocation is only used with lazy region allocation, to be explained in Section 4). If no token is currently available, the GLOBAL one is returned. The FREEREGION operation has to check the DIRTY field in the region descriptor. Only when the region is clean, can pages be reclaimed by adding them to the free list. Otherwise, pages are appended to the page list of the GLOBAL region.

As outlined in Figure 3, the allocator provides two services for write barriers. The CHECKWRITE service is called before 


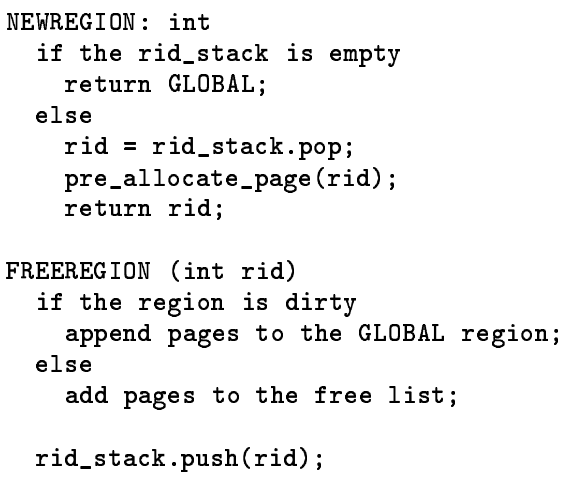

Figure 2: Services for regions

putfield and aastore byte codes. The addresses $l$ hs and $r h s$ point to the left hand side and right hand side objects. The operation filters out null pointers and escaped objects first, then computes page indexes from object addresses and tests equality. Region IDs are retrieved from page descriptors and compared if the objects are not in the same page. The $r h s$ object is marked as escaped if it is not in the region of the lhs object.

The write barrier for putstatic calls MARKESCAPED directly. As we explained in Section 2, the allocator uses a bit vector to record the states of allocation sites. Both services not only mark the region as dirty, but also set the state of the allocation site to non-local. In the object header, a bit in the status word is used to mark an object as escaped.

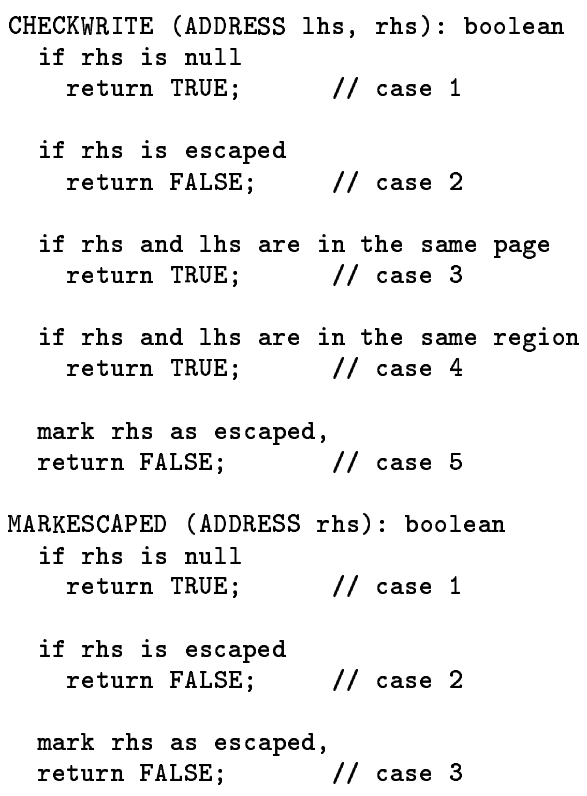

Figure 3: Services for barriers

The main function of the allocator is to allocate space for an object. With regions, the allocation of space is somewhat complicated. The allocation process ALLOC is illustrated in figure 4. Here, we present only a high-level abstraction of the service. The allocation method first checks the state of the allocation site. Only local sites are eligible for allocation from local regions. The internal method _getHeapSpace allocates space in the first page if the free space is larger than the required size. If the first attempt fails, it looks at pages following the current page. If the request cannot be satisfied from these pages, it then looks for contiguous pages by scanning the bit maps. This is the most expensive operation in a region-based allocator.

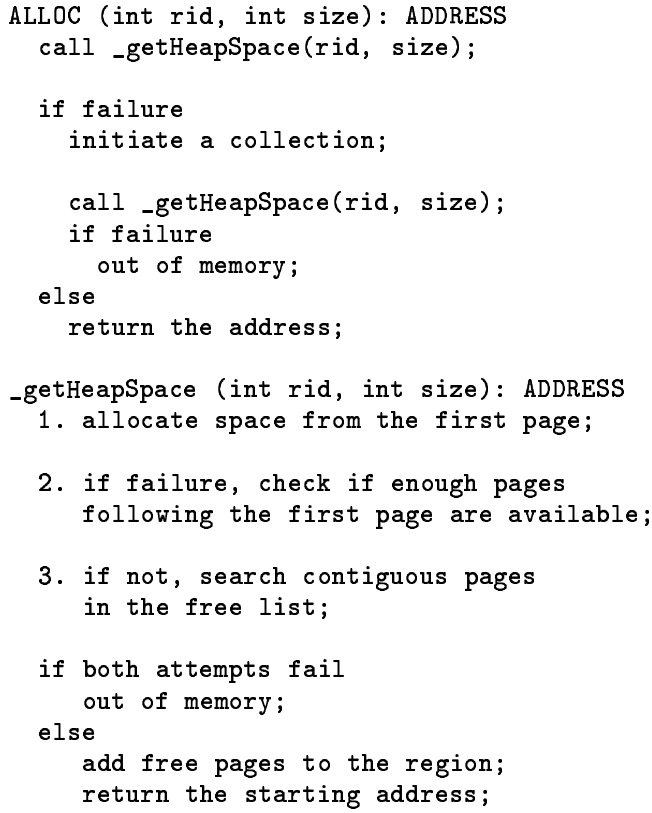

Figure 4: Allocating spaces

These services also provide the facilities required by the garbage collector to perform collections. We discuss the collection process in Section 5.

\section{ADAPTIVE VM}

To utilize regions, a JVM needs the following modifications:

1. Each allocation site is assigned a unique index at compilation time.

2. The object header has a field for recording the index of its allocation site.

3. The stack frame has a slot for the region ID at a fixed offset from the frame pointer.

4. The method prologue and epilogue have additional instructions to deal with the region ID slot.

5. Write barriers are inserted before putstatic, putfield, and aastore byte codes.

The allocation method has two more parameters than before: the index of an allocation site is a runtime constant, and the region ID is fetched from the stack frame.

When deciding whether or not a method is eligible for a new local region, our implementation uses following criteria:

- A native call is assigned the Global region id. 


\begin{tabular}{|l|l|l|l|l|}
31 & \multicolumn{2}{c}{9} \\
\hline$?$ & thread ID & count & & hash code \\
\hline
\end{tabular}

(a) Structure of the status word

\begin{tabular}{|l|l|l|l|}
\hline 0 & non-zero site & & 0 \\
\hline
\end{tabular}

(b) Status of a non-escaping object

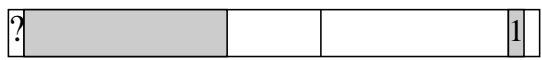

(c) Status of an escaped object

Figure 5: Sharing bits with thin locks

- The <clinit> method always uses the GLOBAL region since we know that it initializes static fields.

- The <init $>$ method inherits the caller's region because it initializes the instance fields.

- A method returning an object is not eligible for a new region. This rule eliminates the need for a write barrier for the areturn byte code. More importantly, as pointed out by [8], there are many methods just allocating objects for the caller.

- A one-pass scan of the byte codes counts the allocation sites of each method. If the number is lower than a threshold, no local region is needed for this method. We currently use a threshold of 1 .

- The first executed method of the booting thread is assigned the GLOBAL region ID.

In our initial development it became clear that making newregion and freeregion calls on each activation is too expensive for the run time system since many activations may have empty regions, either because their allocation sites have become non-local or because no object is allocated. To eliminate these empty regions, we use lazy region allocation. An eligible method first saves a special region ID, e.g. 0, in the region ID slot, indicating the stack frame needs a dynamic region, but it has not yet been allocated. The code for allocation first checks the ID, and then calls newregion only when necessary. The freeregion method is called only when the region ID is a valid one. If a method inherits a region from its caller, it must write back its current region ID to the caller's stack frame.

Another implementation issue is how to encode the allocation site index in the object header. A two-word object header is quite a popular design on most JVMs. One word of the header is used as a status word. Our implementation avoids growing the object header by storing the allocation site index in space already used by the thin lock [3].

In the Jikes RVM, the thin lock uses 13 bits for recording the ID of the locking thread, and 6 bits for counting locks. Figure 5(a) shows the structure of the status word. Bit 31 is called the monitor shape bit which is 0 if the lock is thin and 1 if it is fat.

As indicated in Figures 5 (b) and (c), we use bit 1 in the status word to indicate if the object has escaped or not ${ }^{2}$. If

\footnotetext{
${ }^{2}$ Bit 1 is used for write barrier purpose in other types of GC. In our prototype implementation in a copying collector, this bit is used as the escaping bit.
}

the object is non-escaping, then we reuse the thread ID field to store the allocation site (Figure $5(\mathrm{~b})$ ). This reuse of the thread ID field necessitates some extra machinery for the case where a lock operation is performed on a non-escaping object. The thin lock mechanism first attempts to check the monitor shape bit and the thread ID field in the status word. In ordinary thin locks, the common case is that the monitor shape bit and the thread ID are both zero. However, in our scheme, a non-escaping object is using the thread ID field and it will be non-zero. Thus, when a thin lock fails we must check to see if it failed because a non-escaping object is reusing the thread ID field. If the object is non-escaping, we give back the field to the thin lock by clearing the thread ID field, setting the escaping bit, and then attempting the lock operation again.

By changing a non-escaping object to escaping, we do lose some opportunities for finding local objects, but we do not affect the behaviour of the thin locks. In Section 6.5 we show that this effect is not too large. To ensure correctness of this scheme, an escaped object must never become nonescaping, and whenever an object is marked as escaped, the associated region must be marked as dirty.

The system only adds a check on the uncommon path of the thin lock and may need one check on the common path in very few cases. The mechanism allows us to encode allocation site numbers up to $2^{13}-1$. For large applications, it would be possible to use both the thread ID and lock count to store a 19-bit allocation site index if their positions were reversed (to ensure that small allocation site index still produces a non-zero thread ID field).

There are some other issues related to Java semantics [10]. An exception may transfer control to the caller without going through the method epilogue. In this case, the exception mechanism must release the region before unwinding the stack frame.

If an object has a non-trivial finalize method, the JVM has to run the finalizer before the space is reused. The regionbased allocator organizes the list of objects with non-trivial finalizers by region ID. When the pages of a region are about to be reclaimed, the finalize methods of objects in the region get called.

\section{COLLECTOR}

The collector must ensure that if an object escapes its original region, that region is marked as dirty. One way of ensuring this would be to introduce write barriers during collections. However, this may sacrifice the efficiency of the collector. There is a trade-off between precision and performance. If all live objects are copied to dirty regions, no barriers are needed. So, the second option is to copy all live objects to the GLOBAL region of another space. This does not violate the above rule since the global region never gets released. This strategy has the same efficiency as a normal copying collector. However, copying all objects to the GLOBAL region may cause some objects created in the next epoch to be treated as escaped, and their associated allocation sites marked as non-local, unnecessarily.

Our current implementation keeps objects in their original region as much as possible, and marks all live regions as dirty after the collection. Now objects in the root set are divided into subsets by their regions, with each live region corresponding to a subset of the root set. The collector starts with collecting all reachable objects from the subset belong- 
ing to the Global region. In the next step, the collector collects objects reachable from the subset corresponding to each local region. All objects copied to the GLOBAL region are marked as escaped to allow fast checks in barriers (the states of the allocation sites are not changed). Although this strategy makes some stackable objects in current live regions unstackable, it does not require write barriers and will not make allocation sites non-local unnecessarily. Currently, we do not have experimental evidence to show which option is better in reality.

\section{RESULTS}

We implemented a page-based heap and a prototype of a region-based allocator in the Jikes RVM with the baseline compiler. Porting of the system to the optimizing compiler is in progress. The region-based allocator is implemented in a semi-space copying collector using Cheney's tracing algorithm. The current implementation uses a uniprocessor configuration. However, it can be implemented in existing parallel collectors with little effort.

To understand the program behavior, we did detailed profiling of the allocation behaviors, and report the experimental results of the following aspects:

- the allocation behavior of the region-based allocator;

- the percentage of space reclaimed by local regions, and the reduction in collections due to local region allocation;

- the behavior of write barriers;

- the impact on thin locks; and

- the effect of adaptivity.

\subsection{Benchmarks}

We report experimental results on the SPEC JVM98 benchmark suite and soot-c [15]. The soot-c benchmark is a Java byte code transformation framework that is quite object-oriented, and which has several phases with potentially different allocation behaviors.

We first provide some measurements to give some idea of the allocations performed by each benchmark. Table 1 shows the profiles of allocation sites. The column labeled Compiled gives the total number of allocation sites in compiled methods. It includes the allocation sites in the JVM, libraries and benchmark code. The column labeled Used lists the number and percentage of allocation sites which created at least one object. On average $26 \%$ of the allocation sites create at least one object. The columns labeled Non-local and Local show the fraction of used allocation sites which are categorized as non-local and local. An allocation site is categorized as local if it is never marked as non-local by the adaptive algorithm. The last column, labeled Max $R I D$, gives the maximum number of regions used by the benchmark at the same time. This gives us some idea of the number of region tokens required. Note that a program (like javac) with deep recursion may use a large number of regions.

In all of our experiments the total heap size is set to $50 \mathrm{M}$, from which the JVM uses about $1.5 \mathrm{M}$ as the boot area. The JVM itself shares the same heap with applications. We do not distinguish the objects created by the system or the benchmarks. The heap is divided into two semi-spaces. A
$25 \mathrm{M}$ heap is quite small for most of our benchmarks, which forces the garbage collector to work.

\subsection{Choice of Page Size}

The choice of page size may affect utilization of heap space. A larger page size will allow more allocations to be satisfied in the first page. On the other hand, smaller page size will reduce the amount of froth (unused pieces of the heap due to the allocation of chunks/pages of memory ${ }^{3}$ ). Table 2 shows the the effect of different page sizes on the number of garbage collections needed and the froth rates. The column labeled Base collections gives the number of collections needed for the base semi-space copying garbage collector, without the regions. The three columns labeled clc give the collections required for the region-based allocator, assuming page sizes of 256 bytes, $1 \mathrm{~K}$ bytes and $4 \mathrm{~K}$ bytes. ${ }^{4}$ Similarly, the columns labeled froth give the wasted space for the three different page sizes (computed by unused_bytes/allocated_bytes). Note that a page size of $4 \mathrm{~K}$ leads to a large froth rate for several benchmarks, most notably javac (130\%), jack (27.5\%) and soot-c (23.5\%). The very high froth rate for javac also seems to increase the number of garbage collections, which is more than double that of the base collector.

From the perspective of number of collections and froth, the smaller pages seem better. However, this is not the complete story. One must also consider the overhead for allocations. The cheapest form of allocation is when the newly allocated object fits in the current page, the second cheapest is when the allocation can be allocated on the next page, and the most expensive is when one must search the free list for enough contiguous pages to meet the allocation request. These overheads are summarized in Table 3 . Considering the three page size configurations: 256-byte, $1 \mathrm{~K}$, and $4 \mathrm{~K}$, the allocations are categorized into three types, which reflect three possibilities in _getHeapSpace in Figure 3.

1. firstpage The space is available in region's first page.

2. nextpages The region is expanded with immediately contiguous pages.

3. searching Search for contiguous pages in the free list.

A large size $(4 \mathrm{~K})$ allows most of allocations to be satisfied with cheap costs. But, as we demonstrated in Table 2 , the froth rate may run out of control. From Table 3 we see that there is not a large difference in the behavior of allocations when comparing page sizes of $1 \mathrm{~K}$ and $4 \mathrm{~K}$. However, even though a smaller page size (256 bytes) reduces froth rates, the allocation distribution changes dramatically, with many more allocations requiring expensive operations. From these results we conclude that the trade-off between page size and froth rate is worth considering when using a page-based heap. For our remaining experiments we chose a page size of $1 \mathrm{~K}$, which gives us both reasonable froth rate and reasonable allocation overhead.

\subsection{Region-reclaimed space}

The next important measurement is to find out the percentage of space that is reclaimed from local regions. That is, how much space can be reclaimed when using the regionbased approach. Recall that the region can be reclaimed

\footnotetext{
${ }^{3}$ This term was introduced by Steensgaard [12].

${ }^{4}$ We disabled System.gc() calls for both collectors.
} 


\begin{tabular}{|l|c|r|r|r|c|}
\hline Benchmark & Compiled & Used & Non-local & Local & Max RID \\
\hline compress & 2108 & $346(16 \%)$ & $115(33 \%)$ & $231(67 \%)$ & 11 \\
db & 2117 & $358(16 \%)$ & $124(34 \%)$ & $234(66 \%)$ & 11 \\
jack & 2396 & $614(25 \%)$ & $204(33 \%)$ & $410(67 \%)$ & 16 \\
javac & 2871 & $895(31 \%)$ & $437(48 \%)$ & $458(52 \%)$ & 56 \\
jess & 2407 & $577(23 \%)$ & $276(47 \%)$ & $301(53 \%)$ & 9 \\
mpegaudio & 3266 & $1502(45 \%)$ & $157(10 \%)$ & $1345(90 \%)$ & 12 \\
mtrt & 2228 & $497(22 \%)$ & $196(39 \%)$ & $301(61 \%)$ & 19 \\
soot-c & 3030 & $1158(34 \%)$ & $551(52 \%)$ & $507(48 \%)$ & 14 \\
\hline
\end{tabular}

Table 1: Allocation sites

\begin{tabular}{|l|c||r|r||r|r||r|r|}
\hline \multirow{2}{*}{ Benchmark } & \multicolumn{1}{|c||}{ Base \# } & \multicolumn{2}{c||}{ 256 Bytes } & \multicolumn{2}{c||}{ 1K Bytes } & \multicolumn{2}{|c|}{ 4K Bytes } \\
\cline { 2 - 7 } & collections & clc & froth & clc & froth & clc & froth \\
\hline compress & 7 & 7 & $0.03 \%$ & 7 & $0.11 \%$ & 7 & $0.47 \%$ \\
db & 4 & 4 & $0.05 \%$ & 4 & $0.23 \%$ & 4 & $1.05 \%$ \\
jack & 9 & 7 & $1.29 \%$ & 8 & $5.97 \%$ & 9 & $27.52 \%$ \\
javac & 12 & 12 & $4.96 \%$ & 15 & $29.41 \%$ & 25 & $130.42 \%$ \\
jess & 12 & 11 & $0.13 \%$ & 11 & $0.53 \%$ & 11 & $2.19 \%$ \\
mpegaudio & 0 & 0 & $0.62 \%$ & 0 & $2.10 \%$ & 0 & $9.05 \%$ \\
mtrt & 7 & 1 & $0.03 \%$ & 1 & $0.09 \%$ & 1 & $0.38 \%$ \\
soot-c & 15 & 13 & $1.09 \%$ & 13 & $4.89 \%$ & 15 & $23.49 \%$ \\
\hline
\end{tabular}

Table 2: Effect of page size on \# of collections and froth

\begin{tabular}{|l|r|r|r|r|}
\hline Benchmark & $\begin{array}{r}\text { page } \\
\text { size }\end{array}$ & firstpage & nextpages & searching \\
& 256 & $82.73 \%$ & $16.23 \%$ & $1.04 \%$ \\
\hline compress & $1 \mathrm{~K}$ & $94.96 \%$ & $4.74 \%$ & $0.30 \%$ \\
& $4 \mathrm{~K}$ & $98.43 \%$ & $1.44 \%$ & $0.13 \%$ \\
\hline $\mathrm{db}$ & 256 & $92.24 \%$ & $7.69 \%$ & $0.07 \%$ \\
& $1 \mathrm{~K}$ & $98.04 \%$ & $1.92 \%$ & $0.03 \%$ \\
& $4 \mathrm{~K}$ & $99.49 \%$ & $0.48 \%$ & $0.03 \%$ \\
\hline jack & 256 & $91.64 \%$ & $6.46 \%$ & $1.91 \%$ \\
& $1 \mathrm{~K}$ & $97.79 \%$ & $1.59 \%$ & $0.63 \%$ \\
& $4 \mathrm{~K}$ & $99.48 \%$ & $0.33 \%$ & $0.19 \%$ \\
\hline javac & 256 & $89.56 \%$ & $8.58 \%$ & $1.85 \%$ \\
& $1 \mathrm{~K}$ & $97.43 \%$ & $2.00 \%$ & $0.57 \%$ \\
& $4 \mathrm{~K}$ & $99.41 \%$ & $0.50 \%$ & $0.09 \%$ \\
\hline jess & 256 & $86.75 \%$ & $12.89 \%$ & $0.36 \%$ \\
& $1 \mathrm{~K}$ & $96.71 \%$ & $3.27 \%$ & $0.02 \%$ \\
& $4 \mathrm{~K}$ & $99.16 \%$ & $0.83 \%$ & $0.01 \%$ \\
\hline mpegaudio & 256 & $84.97 \%$ & $12.09 \%$ & $2.94 \%$ \\
& $1 \mathrm{~K}$ & $95.54 \%$ & $3.52 \%$ & $0.94 \%$ \\
& $4 \mathrm{~K}$ & $98.59 \%$ & $0.98 \%$ & $0.43 \%$ \\
\hline mtrt & 256 & $96.04 \%$ & $2.58 \%$ & $1.39 \%$ \\
& $1 \mathrm{~K}$ & $99.51 \%$ & $0.38 \%$ & $0.11 \%$ \\
& $4 \mathrm{~K}$ & $99.88 \%$ & $0.10 \%$ & $0.02 \%$ \\
\hline soot-c & 256 & $88.28 \%$ & $9.85 \%$ & $1.88 \%$ \\
& $1 \mathrm{~K}$ & $96.85 \%$ & $2.66 \%$ & $0.49 \%$ \\
& $4 \mathrm{~K}$ & $99.21 \%$ & $0.68 \%$ & $0.11 \%$ \\
\hline
\end{tabular}

Table 3: Behaviors of allocations

when a stack activation is popped only when the dirty bit has not been set (i.e. the region is clean). If any object in the region has escaped, then the dirty bit will be set, and this region must be added to the GLOBAL region which will be collected by the garbage collector.
The table given in Figure 6(a) gives the bytes reclaimed from clean regions and the percentage they represent of total allocated bytes, when the page size is $1 \mathrm{~K}$. Different page sizes give very similar numbers. The percentage of region-reclaimed bytes varies between benchmarks. In the best case, mtrt has 80 percent of total allocated memory reclaimed by regions, with the number of collections reduced from 7 to 1 . In the worst case, $d b$ has less than $1 \%$ regionreclaimed space, with no impact on the number of collections.

Another way to look at the behavior of the regions is to examine the number of bytes allocated from local regions over the duration of the execution. Figure $6(\mathrm{~b})$ shows the fraction of bytes allocated that are allocated from local regions. The $\mathrm{x}$-axis is an abstraction of time, with each unit corresponding to $1 \mathrm{M}$ bytes of allocations. The $\mathrm{y}$-axis shows the fraction of those $1 \mathrm{M}$ bytes that were allocated from a local region. For example, the graph labeled mtrt indicates that after an initial startup, about $90 \%$ of all allocated bytes are allocated from local regions. In contrast, the graph labeled jess shows that this benchmark quickly declines to less than $10 \%$ of all allocations from local regions. The graph labeled soot-c shows a widely fluctuating rate as the program progresses. This is likely because soot-c is quite a complex benchmark with many different phases. It is interesting to note that if all the bytes allocated to local regions are also region-released, then the area under the curves of Figure 6(b) should be equal to the region-reclaimed number shown in Figure 6(a). This appears to be the case, as confirmed by our measurements given in Section 6.6, showing that almost all local regions are clean when released.

\subsection{Write Barrier Behavior}

Another important aspect of the collector we measured is the behavior of write barriers, as summarized in Table 4. Overall, a write barrier has bounded constant time as 


\begin{tabular}{|l|c|c||r|r|r|}
\hline Benchmark & $\begin{array}{c}\text { base } \\
\text { collections }\end{array}$ & $\begin{array}{c}\text { total } \\
\text { allocated }\end{array}$ & region-reclaimed & clc & froth \\
\hline compress & 7 & $116 \mathrm{M}$ & $15.39 \mathrm{M}(13.27 \%)$ & 7 & $0.11 \%$ \\
db & 4 & $77 \mathrm{M}$ & $0.57 \mathrm{M}(0.74 \%)$ & 4 & $0.23 \%$ \\
jack & 9 & $223 \mathrm{M}$ & $51.00 \mathrm{M}(22.87 \%)$ & 8 & $5.97 \%$ \\
javac & 12 & $212 \mathrm{M}$ & $18.65 \mathrm{M}(8.80 \%)$ & 15 & $29.41 \%$ \\
jess & 12 & $267 \mathrm{M}$ & $17.36 \mathrm{M}(6.50 \%)$ & 11 & $0.53 \%$ \\
mpegaudio & 0 & $7 \mathrm{M}$ & $1.96 \mathrm{M}(28.00 \%)$ & 0 & $2.10 \%$ \\
mtrt & 7 & $143 \mathrm{M}$ & $115.15 \mathrm{M}(80.52 \%)$ & 1 & $0.09 \%$ \\
soot-c & 15 & $219 \mathrm{M}$ & $40.72 \mathrm{M}(18.59 \%)$ & 13 & $4.89 \%$ \\
\hline
\end{tabular}

(a) Region-reclaimed space

javac (per 1M)

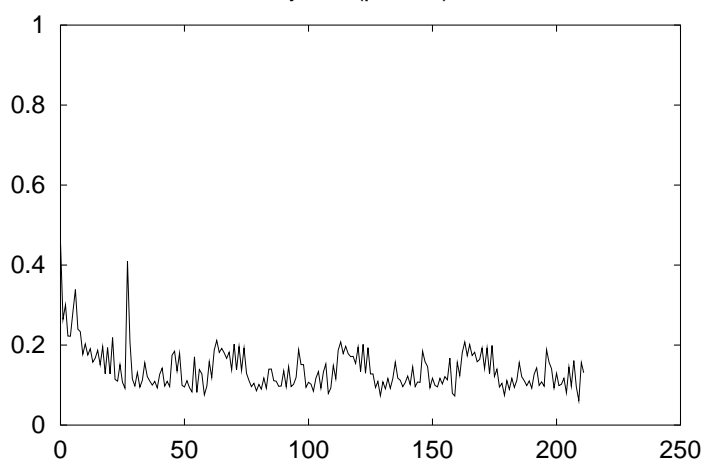

mtrt (per 1M)

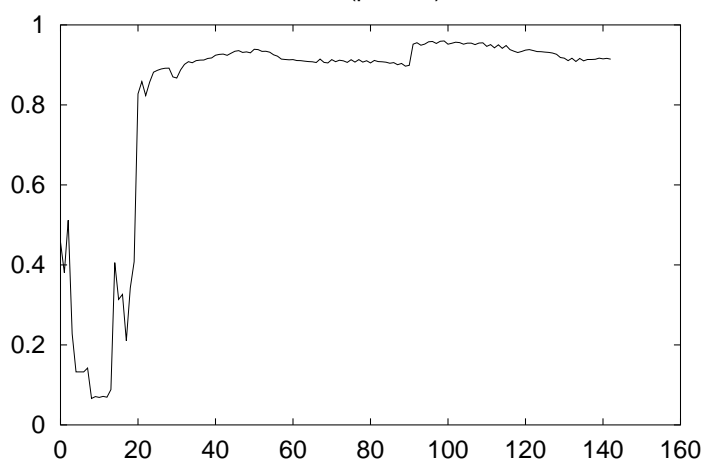

jess (per 1M)

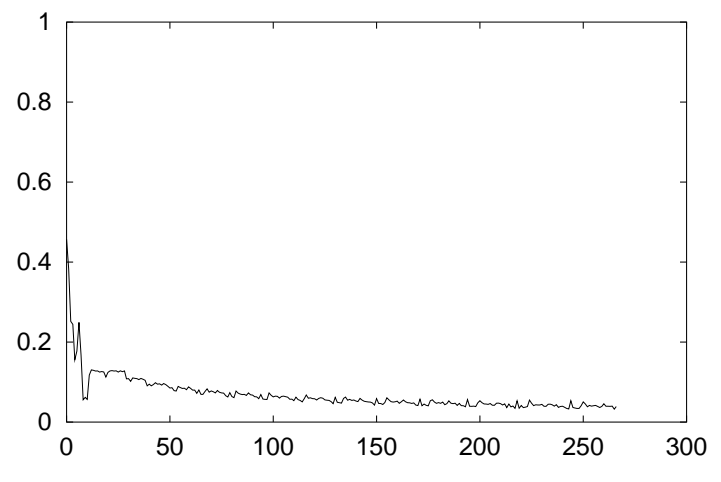

soot-c (per 1M)

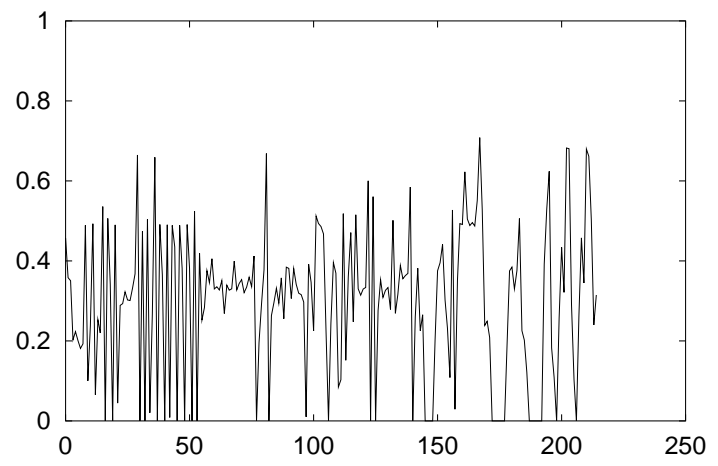

(b) Bytes allocated in local regions

Figure 6: Behavior of Local Regions

\begin{tabular}{|l|r|r|r|r|r||r|r|r|}
\hline BENCHMARK & \multicolumn{4}{|c||}{ CHECKWRITE for putfield and aastore } & \multicolumn{3}{|c|}{ MARKESCAPED for putstatic } \\
\hline & null & quick & samepage & sameregion & escaped & null & quick & escaped \\
\hline compress & $15.07 \%$ & $83.64 \%$ & $1.05 \%$ & $0.17 \%$ & $0.07 \%$ & $0.00 \%$ & $91.45 \%$ & $8.55 \%$ \\
db & $0.12 \%$ & $99.88 \%$ & $0.00 \%$ & $0.00 \%$ & $0.00 \%$ & $0.00 \%$ & $90.48 \%$ & $9.52 \%$ \\
jack & $11.79 \%$ & $87.58 \%$ & $0.62 \%$ & $0.01 \%$ & $0.00 \%$ & $0.00 \%$ & $91.00 \%$ & $9.00 \%$ \\
javac & $10.81 \%$ & $88.84 \%$ & $0.32 \%$ & $0.03 \%$ & $0.00 \%$ & $2.90 \%$ & $89.63 \%$ & $7.47 \%$ \\
jess & $0.22 \%$ & $99.75 \%$ & $0.02 \%$ & $0.00 \%$ & $0.00 \%$ & $1.88 \%$ & $85.63 \%$ & $12.50 \%$ \\
mpegaudio & $0.42 \%$ & $98.12 \%$ & $0.08 \%$ & $1.38 \%$ & $0.00 \%$ & $3.20 \%$ & $83.56 \%$ & $13.24 \%$ \\
mtrt & $13.82 \%$ & $84.49 \%$ & $1.66 \%$ & $0.02 \%$ & $0.00 \%$ & $0.00 \%$ & $86.44 \%$ & $13.56 \%$ \\
soot-c & $5.20 \%$ & $91.88 \%$ & $2.71 \%$ & $0.21 \%$ & $0.00 \%$ & $0.00 \%$ & $93.48 \%$ & $6.52 \%$ \\
\hline
\end{tabular}

${ }^{*}$ In this table, zero only means the rate is lower than $0.005 \%$.

Table 4: Behaviors of write barriers 
shown by the pseudo-code in Figure 3. However, it is still a burden to the system. To get better idea of how to optimize the barriers, we categorize the CHECKWRITE for putfield and aastore into five types.

1. the right hand side is a null pointer,

2. the right hand side object is already marked as escaped,

3. both objects are in the same page,

4. both objects are in the same region, and

5. the $L H S$ and $R H S$ objects are not in the same region.

The first case checks if the right hand side reference is a null pointer, and the second case checks the escaping bit, which requires a load and a compare instruction. As shown in Table 4, the majority of checks are filtered out by these two cases, which indicates it is beneficial to separate these two cases as a common path and inline them. The remaining three cases can be processed by a method call. Similarly the write barriers for putstatic are also categorized into three types, with the first two cases benefiting from inlining.

Currently, we have not incorporated any static analysis for removing write barriers. Certainly, such analyses will reduce the runtime cost of the system. We will investigate such algorithms in the future.

\subsection{Impact on Thin Locks}

We also profiled the impact of sharing bits with thin locks. Table 5 shows the rate of failed locks because of sharing bits. Compress and mpegaudio have only a few thousand locks in a full run, so their results cannot represent the real effect of sharing bits. On other benchmarks, the rate of spoiled locks is no more than $5 \%$.

\begin{tabular}{|l|r|r|}
\hline Benchmark & thin locks & spoiled locks \\
\hline compress & $1.6 \mathrm{~K}$ & $172(9.58 \%)$ \\
db & $45.2 \mathrm{M}$ & $2915(0.01 \%)$ \\
jack & $9.4 \mathrm{M}$ & $497016(5.00 \%)$ \\
javac & $14.7 \mathrm{M}$ & $341585(2.26 \%)$ \\
jess & $4.8 \mathrm{M}$ & $4881(0.10 \%)$ \\
mpegaudio & $5.6 \mathrm{~K}$ & $497(8.15 \%)$ \\
mtrt & $1.3 \mathrm{M}$ & $25816(1.92 \%)$ \\
soot-c & $5.6 \mathrm{M}$ & $73204(1.30 \%)$ \\
\hline
\end{tabular}

Table 5: Impact on thin locks

\subsection{Effect of Adapting}

The last behavior that we studied was the effect of the adaptive part of our algorithm. The basic idea of our approach was to mark allocation sites as non-local as soon as they are found escaping the first time. The justification of this decision was that this would prevent this allocation site from spoiling clean regions in the future, and we expected that this would lead to most local regions being clean at release time. Figure 7(a) shows the number of local regions that are clean at release time over the duration of the execution of the program. The $\mathrm{x}$-axis is an abstraction of time, with each point representing the release of 1000 local regions (100 for jess). The y-axis shows the number of those local regions that are clean at release time. Accompanying the title of each graph is the number of clean regions and total allocated regions. It is very clear that after a short startup time, the system quickly adapts so that almost $100 \%$ of the local regions are clean at release time. There is occasionally a small dip, but then the system adjusts and it goes back to almost $100 \%$. So, it does appear that the system adapts well.

In order to see what would happen without adapting, we removed the part of the algorithm that marks an allocation site as non-local, so that all $^{5}$ allocations are placed in the local regions. Figure $7(\mathrm{~b})$ shows the result in this case. First, note that many more regions are created, and the scale on these graphs are now per 10000 local regions (1000 for jess). However, we can also see some interesting trends. The benchmark mtrt appears to create mostly nonescaping objects, so for this benchmark it is not such a bad idea to just put all objects in local regions. For benchmarks javac and soot-c, we see that removing the adaption leads to many more regions, and many of those regions are not clean. In these cases the adaption works to cull those dirty regions. For jess we see a very interesting behavior, in that in the last two thirds of the execution, a lot of objects appear to be non-escaping, and the number of clean regions stays quite high. With the adaption, we get a higher percentage of clean regions, but we don't find nearly as many. In this case we suspect that there are some allocation sites which sometimes produce escaping objects, and sometimes not. A more complicated prediction scheme appears to be necessary for this kind of benchmark.

We also collected our overall measurements for the two cases, with adaption and without adaption. These are summarized in Table 6 . Note that in some cases, most notably javac, switching off the adaption drastically increases the froth rate (589\% instead of $29 \%$ ) and number of garbage collections (96 instead of 15). However, as we might have predicted from the graph in Figure 7(b), the performance for jess is much better without adaption. This is a clear sign that we must look at other forms of adaption that are more robust when the objects created from a site sometimes escape, and sometimes do not. Overall, it seems that the adaptive algorithm gives better performance and controls excessive froth.

The third column of Table 6 gives the total size of escaping objects which were captured by write barriers or reachable from escaping objects. Locked objects are also considered as escaping. The difference between the total allocated size and the size of escaping objects gives us a rough upper bound of the space that can be reclaimed by regions. We see that there is a large space to improve the current prediction scheme.

\subsection{Summary}

Our current implementation, using the baseline compiler, was aimed at producing a prototype that could be used to measure the behavior of the system, as we have presented in this section. Our numbers show that: 1) page size is important, but with the appropriate page size, the overhead for froth and the frequency of expensive searches for free pages is quite low; 2) for many benchmarks a significant percentage of allocated memory can be placed in local regions which are still clean at release time; 3) appropriate choices for the barrier operations can put the common cases on a low-cost

\footnotetext{
${ }^{5}$ except native calls, <clinit $>$, and the first executed method of the boot thread, see Section 4 .
} 
javac (per 1000), 498K/499K

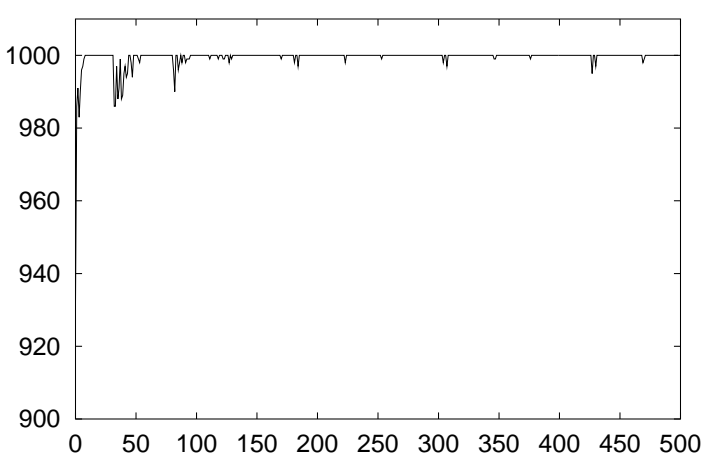

jess (per 100), 72K/72K

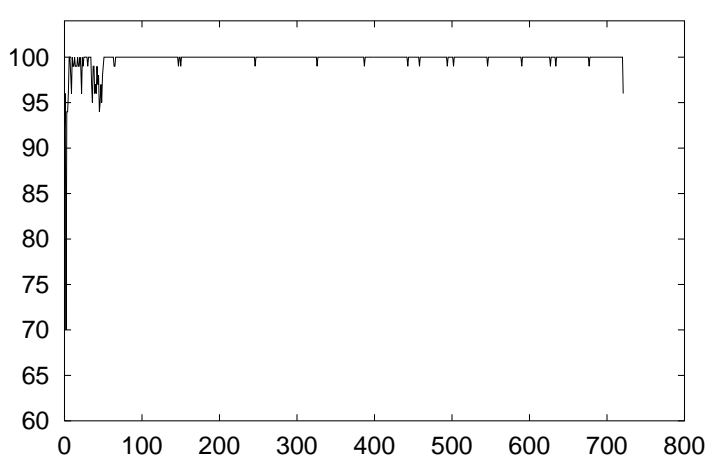

mtrt (per 1000), 2.96M/2.96M

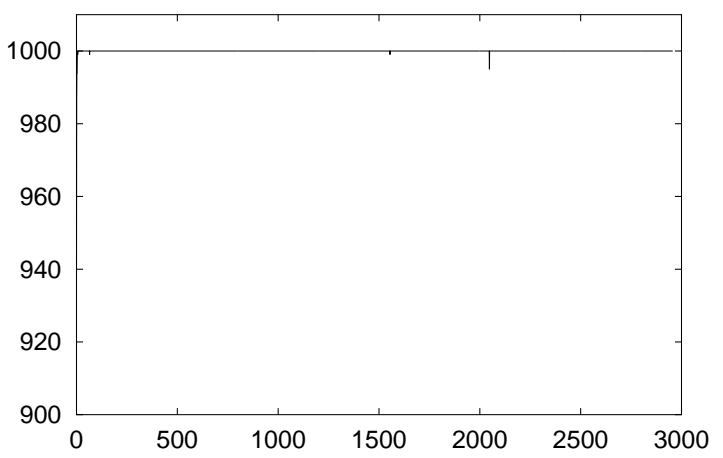

soot-c (per 1000), 406K/407K

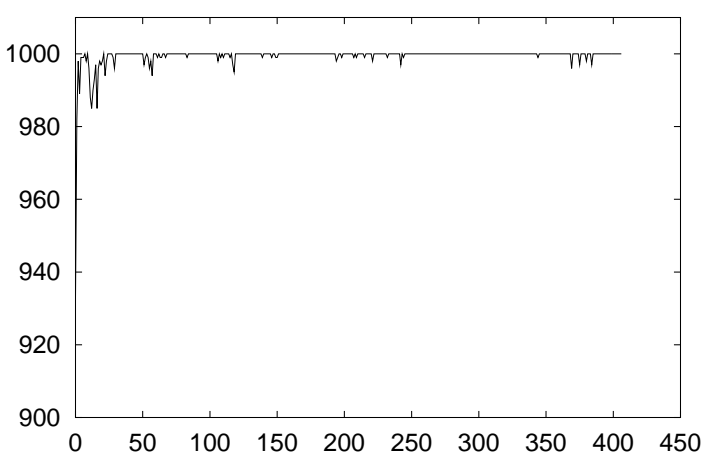

(a) With adaption javac (per 10000), 449K/1.82M

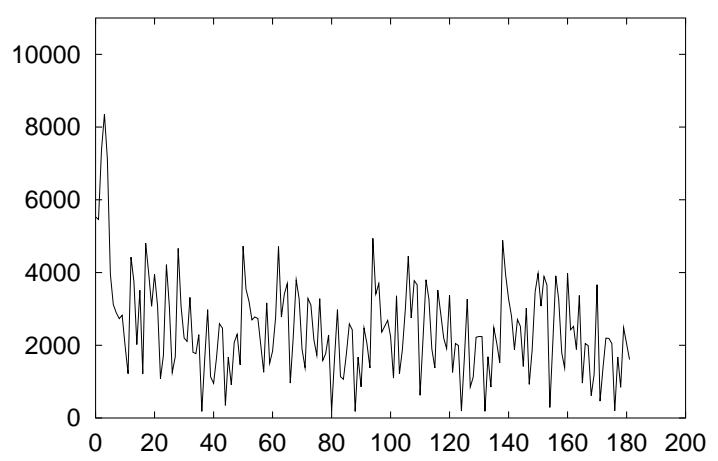

jess (per 1000), 117K/149K

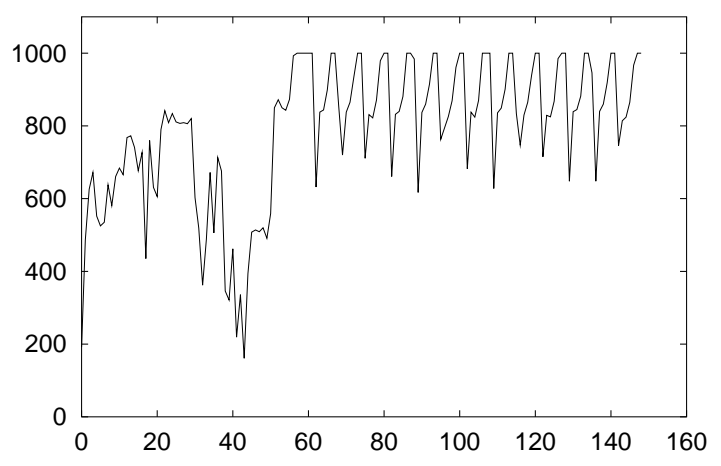

mtrt (per 10000), 2.9M/3.04M

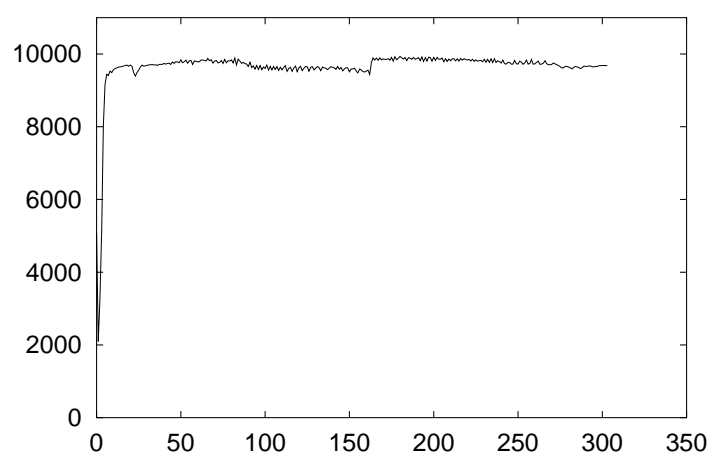

soot-c (per 10000), 419K/1.07M

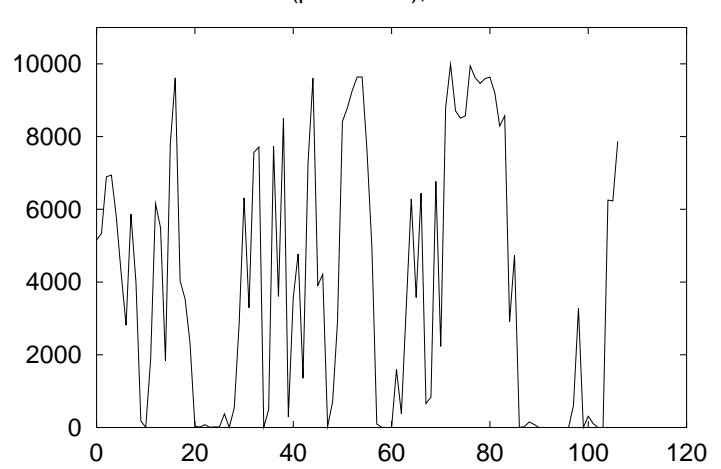

(b) No adaption

Figure 7: Clean regions released 


\begin{tabular}{|c|c|c|c|c|c|c|c|c|c|}
\hline \multirow[b]{2}{*}{ Benchmark } & & & & \multicolumn{3}{|c|}{ With Adaption } & \multicolumn{3}{|c|}{ No Adaption } \\
\hline & $\begin{array}{r}\text { total } \\
\text { allocated }\end{array}$ & $\begin{array}{r}\text { total } \\
\text { escaped }\end{array}$ & $\begin{array}{r}\text { base } \\
\text { clc }\end{array}$ & $\begin{array}{l}\text { region } \\
\text { reclaimed }\end{array}$ & clc & froth & $\begin{array}{c}\text { region } \\
\text { reclaimed }\end{array}$ & clc & froth \\
\hline & $116 \mathrm{M}$ & $99 \mathrm{M}$ & 7 & $15.39 \mathrm{M}(13.27 \%)$ & 7 & $0.11 \%$ & $14.75 \mathrm{M}(12.72 \%)$ & 7 & $3.61 \%$ \\
\hline & & & 4 & $74 \%)$ & 4 & $0.23 \%$ & $M(0.68 \%)$ & 4 & $5.77 \%$ \\
\hline & & & 9 & 51 & 8 & $5.97 \%$ & M $(42.45 \%)$ & 7 & $16.26 \%$ \\
\hline & & $112 \mathrm{M}$ & 12 & $5 \mathrm{M}(8.80 \%)$ & 15 & $29.41 \%$ & $\mathrm{LM}(11.51 \%)$ & 96 & $589.09 \%$ \\
\hline & & & 12 & & 11 & & $\mathrm{M}(84.24 \%)$ & 2 & $9.04 \%$ \\
\hline & $7 \mathrm{M}$ & & 0 & 4.280 & 0 & & $1.37 \mathrm{M}(19.57 \%)$ & 0 & $128.43 \%$ \\
\hline metrt & & $17 \mathrm{M}$ & 7 & $115.15 \mathrm{M}(80.52 \%)$ & 1 & $0.09 \%$ & $112.56 \mathrm{M}(78.71 \%)$ & 6 & $62.42 \%$ \\
\hline soot-c & $219 \mathrm{M}$ & $89 \mathrm{M}$ & 15 & $40.72 \mathrm{M}(18.59 \%)$ & 13 & $4.89 \%$ & $7.82 \mathrm{M}(3.57 \%)$ & 57 & $276.54 \%$ \\
\hline
\end{tabular}

\section{Table 6: Effect of Adaption}

path; 4) the overhead for sharing space with thin locks seems acceptable; 5) the adaptive part of the algorithm is important for focusing the system on the allocation sites that are likely not to escape; and 6) for many, but not all, benchmarks the adaptive system finds more local regions than a non-adaptive system.

We did try measuring runtime improvement using this prototype, but it turned out that the overheads in our current implementation are still too high, and this can lead to an overall slow down. This is due to several factors, including: 1) the cost of region management, 2) the cost of write barriers, and 3) small helper methods used by region and barrier implementation. Since many of these functions are currently written in Java, using small methods, we expect that the optimizing compiler will inline aggressively and optimize away much of the overhead. We are currently working on this implementation in order to determine if the overheads can be reduced to an acceptable level.

\section{RELATED WORK}

We have described a region-based allocator using pagebased heaps. Although we use the terminology region here, the technique does not involve any region inference algorithm [14]. The technique provides an alternative way to allocate objects on stack in a JVM. There is much literature on garbage collection, region-based memory management and object stack-allocation, thus we focus on those systems most closely related to our work.

Tofte's region inference system $[14,13]$ automatically infers regions for objects. It achieves automatic memory management by compiler analyses. Gay and Aiken's C@ [6] and $\mathrm{RC}$ [7] provide language support for regions. C@ does not require an inference algorithm. It uses reference counting and stack scans to determine the safety of reclaiming a region. The main point of our work was to develop a system that works for an existing language, Java, and that is transparent to the Java programmer. Steensgaard [12] proposed threadspecific heaps for multi-threaded programs. Both systems require the heap to be organized as pages/chunks. We studied the allocation behaviors of Java programs on page-based heaps. The preliminary results suggest that Java programs are sensitive to the page size.

Escape analyses [5, 4, 16, 8] for Java determine whether the objects created by an allocation site may escape certain scopes. Mainly the analysis results can be used in two optimizations. Thread escape analysis results can be used to remove unnecessary synchronizations, and escape analy- sis to find method-bounded allocation sites can be used to create objects on the local stack frames. However, the cost of the analyses prevents them from being used at run time, and Java semantics may pose restrictions on stackable objects. Our region-based allocator aims to reduce the work of garbage collector by allocating objects in temporary regions. The technique needs no analyses and may be suitable for a run time system like a Java Virtual Machine.

McDowell [11] reported the number of potentially stackable objects in a set of Java benchmarks. Like other escape analyses, McDowell also made the assumption that a compile time algorithm must make a decision for all objects created by an allocation site, although he was using dynamic profiling information to conclude the results. Our system does not require this limitation. An allocation site may create objects in local regions before any of them is found to be escaped. Extensions of our adaption algorithm may also allow allocation sites to become local again, even after being marked as non-local.

Hallenberg [9] introduced garbage collection into individual regions in Tofte's region inference system for the ML Kit. Although our collector has a similar name as his system, the structures are quite different. In his system, the region inference algorithm creates regions, and inside a region, a copying collector collects live objects. The backbone of our system is a garbage collector, and the region is a natural way to extend stack frames. The region organization serves as the basis of adaptive allocation. Interested readers can find the design of Hallenberg's system in [9], chapter 11.

\section{CONCLUSIONS}

We have presented an adaptive, region-based allocator for Java Virtual Machines and studied the allocation behavior of Java programs on page-based heaps. The main idea is to detect on-the-fly these allocation sites that do not escape their region, and then manage these allocations in local regions that can be released when the associated stack frame is popped.

We implemented the system using the Jikes RVM baseline compiler and associated garbage collector, and we used this prototype to study the behavior of a collection of Java benchmarks, including the SPEC JVM98 benchmarks. This study showed that the design of the system is crucial, including an appropriate choice of page size, and techniques for minimizing space overhead and region allocation/deallocation overhead. We also studied the adaptive mechanism of our 
system, and found that it quickly found regions from which no object escaped.

Given our encouraging results so far, we are currently pushing this work forward in several directions. First, we are porting the system to the optimizing compiler, where we hope that the overhead of region-management and write barriers can be reduced due to aggressive inlining and other compiler optimizations. This will allow us to provide meaningful run-time speedups. Our quantitative measurements of the behavior of the regions and barriers indicate that this should be possible.

Our second major area of investigation is to look at a wider range of adaptive mechanisms. Currently we mark an allocation site as non-local as soon as one object allocated from that site escapes. This scheme is a rather coarse and naive prediction scheme. When we turn off the adaptation, this corresponds to a predictor that always predicts that no object will escape. Our experiments show that this second method works well for those programs where, in fact, a large portion of the objects do not escape. We would now like to examine other more complex predictors. For example, we could use more than 1 bit, and only mark an allocation site as non-local after some number of objects escape. We could also increase the granularity of the predictor by associating dirty bits with pages within regions, rather than having 1 bit per region. Another possibility is to reset allocation sites to being local at intervals, for example at collection time or during phase shifts in the program. This may help in the case where the same allocation site is sometimes local and sometimes non-local. As part of this study we also would like to measure the effect of the regions on memory locality.

Our final area of research is to examine the effect of our system when coupled with different garbage collectors. As we pointed out in Section 5, it should be relatively straightforward to incorporate our ideas in a variety of collectors.

\section{ACKNOWLEDGEMENTS}

We would like to thank the Jikes RVM group at IBM T.J. Watson for providing the Jikes RVM which makes our experiment possible. We particularly appreciated Stephen Fink's helpful comments. The GCTk toolkit from University of Massachusetts facilitated our implementation. We also appreciated John Jorgensen's proofreading of this paper.

\section{REFERENCES}

[1] B. Alpern, C. R. Attanasio, J. J. Barton, M. G. Burke, P. Cheng, J.-D. Choi, A. Cocchi, S. J. Fink, D. Grove, M. Hind, S. F. Hummel, D. Lieber, V. Litvinov, M. F. Mergen, T. Ngo, J. R. Russell, V. Sarkar, M. J. Serrano, J. C. Shepherd, S. E. Smith, V. C. Sreedhar, H. Srinivasan, and J. Whaley. The Jalapeño Virtual Machine. IBM Systems Journal, 39(1):211-238, February 2000.

[2] K. Arnold, J. Gosling, and D. Holmes. The Java Programming Language (Third Edition). Addison-Wesley, 2000.

[3] D. F. Bacon, R. B. Konuru, C. Murthy, and M. J. Serrano. Thin Locks: Featherweight Synchronization for Java. In Proceedings of the 1998 ACM SIGPLAN Conference on Programming Language Design and Implementation (PLDI), pages 258-268, June 1998.
[4] B. Blanchet. Escape Analysis for Object Oriented Languages: Application to Java. In Conference on Object-Oriented Programming, Systems, Languages and Applications (OOPSLA'99), pages 20-34, Nov 1999.

[5] J.-D. Choi, M. Gupta, M. J. Serrano, V. C. Sreedhar, and S. P. Midkiff. Escape Analysis for Java. In Conference on Object-Oriented Programming, Systems, Languages and Applications (OOPSLA'99), pages 1-19, Nov 1999.

[6] D. Gay and A. Aiken. Memory Management with Explicit Regions. In Proceedings of the 1998 ACM SIGPLAN Conference on Programming Language Design and Implementation (PLDI), pages 313-323, Montreal, Canada, June 1998.

[7] D. Gay and A. Aiken. Language Support for Regions. In Proceedings of the ACM SIGPLAN '01 Conference on Programming Language Design and Implementation, pages 70-80, 2001.

[8] D. Gay and B. Steensgaard. Fast Escape Analysis and Stack Allocation for Object-based Programs. In Compiler Construction, 9th International Conference (CC 2000), pages 82-93, 2000.

[9] N. Hallenberg. Combining Garbage Collection and Region Inference in The ML Kit, 1999. Master's thesis. Department of Computer Science, University of Copenhagen, Denmark.

[10] T. Lindholm and F. Yellin. The Java Virtual Machine Specification. Addison-Wesley, 1996.

[11] C. McDowell. Reducing garbage in Java. http://www.cse.ucsc.edu/research/embedded/pubs/gc/.

[12] B. Steensgaard. Thread-Specific Heaps for Multi-Threaded Programs. ACM SIGPLAN Notices, $36(1): 18-24$, January 2000.

[13] M. Tofte. A Brief Introduction to Regions. $A C M$ SIGPLAN Notices, 34(3):186-195, 1999.

[14] M. Tofte and J.-P. Talpin. Region-Based Memory Management. Information and Computation, 132(2):109-176, 1997.

[15] R. Vallée-Rai, E. Gagnon, L. Hendren, P. Lam, P. Pominville, and V. Sundaresan. Optimizing Java bytecode using the Soot framework: Is it feasible? In Compiler Construction, 9th International Conference (CC 2002), pages 18-34, 2000.

[16] J. Whaley and M. Rinard. Compositional Pointer and Escape Analysis for Java Programs. In Conference on Object-Oriented Programming, Systems, Languages and Applications (OOPSLA'99), pages 187-206, Nov 1999. 